\title{
Le système de réparation des mésappariements contrôle la stabilité des télomères
}

Des modifications de l'état fonctionnel des télomères interviennent au moins de deux manières dans le processus de cancérisation. D'abord, l'érosion progressive de l'ADN télomérique lors de la division des cellules crée une source d'instabilité génétique qui conduit normalement à la sénescence, mais peut aussi favoriser l'apparition d'altérations à l'origine du processus de transformation maligne [1]. Par ailleurs, l'acquisition par les cellules tumorales d'une capacité anormale de prolifération nécessite de compenser la perte progressive de l'ADN télomérique [2]. Ces rôles distincts, voire antagonistes, des télomères dépendent évidemment de leur état fonctionnel. Le changement télomérique le mieux connu lors d'un processus tumoral est la réactivation de la télomérase, une enzyme de type transcriptase inverse qui permet de compenser l'érosion des télomères [3]. Son activité est présente dans au moins $85 \%$ des tumeurs étudiées, quelle que soit leur origine, alors qu'elle est absente ou très faible dans la plupart des tissus somatiques normaux [4]. Un mécanisme alternatif à la réactivation de la télomérase, qui permet aussi de maintenir l'ADN télomérique, est appelé ALT (alternative lengthening of telomeres) [5]. Comme l'activation de la télomérase, ce processus est suffisant pour permettre la prolifération à long terme des cellules et, bien que d'apparition moins fréquente, il pourrait jouer un rôle important dans le développement des tumeurs. Les cellules ALT sont dépourvues 1184 des télomères longs et hétérogènes du fait des échanges homologues et non réciproques entre extrémités de chromosomes [6] (figure 1A). Les recombinaisons entre les télomères sont normalement des événements rares dans les cellules, et les cellules ALT ont donc très certainement subi une «activation» dont le mécanisme est inconnu.

V. Lundblad, un chercheur du Baylor College of Medicine (Houston, ÉtatsUnis), a suspecté que le système de réparation des mésappariements de l'ADN (mismatch-repair ou MMR), dont une fonction est d'inhiber les recombinaisons entre séquences imparfaitement homologues (homéologues) [7], pouvait empêcher les recombinaisons entre les télomères et donc l'apparition de cellules ALT. Son équipe a testé cette hypothèse chez la levure du boulanger Saccharomyces cerevisiae qui est capable de préserver la longueur de ses télomères par activation de la télomérase ou par recombinaison [8]. En l'absence de télomérase, la croissance de ces levures ralentit après environ 80 générations (phénotype sénescent). En revanche, des levures à la fois dépourvues de télomérase et mutantes pour un composant du système MMR (msh2, mlh1 ou pms1) ne ralentissent que très légèrement leur prolifération. Ainsi, le blocage du système MMR provoque une inhibition du processus de sénescence [9]. Cet effet n'est pas dû à l'apparition de mutations secondaires à l'instauration d'une instabilité génétique, car la réintroduction expérimentale du gène $\mathrm{MSH} 2$ sauvage est suffisante pour restaurer le processus de sénescence. Par ailleurs, la délétion du gène $R A D 52$, qui est nécessaire au processus de recombinaison ALT, empêche les cellules mutantes pour un composant du système MMR d'échapper à la sénescence. Enfin, les levures déficientes en MMR qui ont échappé à la sénescence présentent les réarrangements chromosomiques caractéristiques des levures ALT. Tous ces résultats convergent donc vers l'idée que le système MMR protège les cellules du processus ALT, probablement en inhibant la recombinaison (figure $1 A$ ).

Plusieurs questions demeurent cependant. Par exemple, comment les intermédiaires de recombinaison télomériques sont-ils reconnus par le système MMR? Chez la levure du boulanger, il est possible que la nature irrégulière des répétitions télomériques soit responsable de mésappariements qui seraient reconnus par ce système de réparation. Cependant, le système MMR confère aussi une protection contre le processus ALT à la levure Kluyveromyces lactis, chez laquelle les répétitions de séquence sont régulières . Dans cet organisme, comme chez l'homme, il existe des variants de séquence de la répétition télomérique dans la partie la plus proximale des télomères (répétitions «dégénérées »), pouvant représenter une source d' «homéologie" suffisante pour être reconnue par le système MMR [10]. Les extrémités des télomères sont normalement protégées des systèmes intervenant dans la surveillance des cassures et la réparation de l'ADN par une 


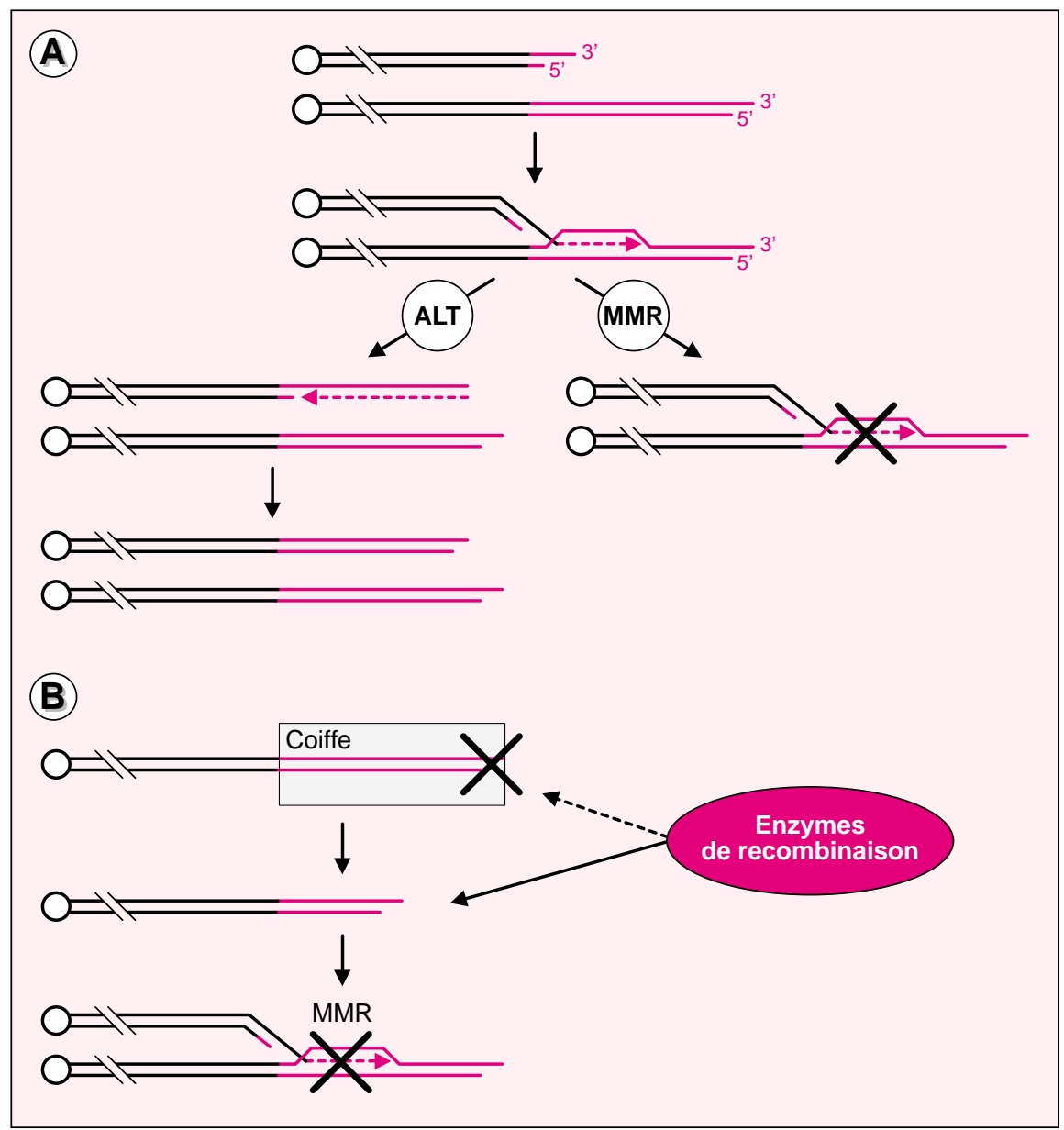

Figure 1. Le système de réparation des mésappariements de l'ADN (MMR) et la recombinaison inter-télomérique. Les répétitions $d^{\prime} A D N$ télomérique sont représentées en rouge. A. Rôle antagoniste des systèmes ALT et MMR dans le maintien de la taille des télomères. Quand la télomérase est absente, le système ALT peut rétablir la longueur d'un télomère très raccourci par recombinaison homologue à travers un échange non-réciproque avec un télomère plus long. Si l'hétéroduplex est reconnu par le système MMR, le processus de recombinaison est inhibé et le télomère court n'est pas allongé. B. Rôles respectifs de la coiffe de protection et du MMR dans l'inhibition des recombinaisons inter-télomériques. La recombinaison est inhibée par des protéines de structure du télomère. Si ces protéines deviennent inactives du fait d'un raccourcissement trop important des télomères, les enzymes de recombinaison ont accès aux extrémités des chromosomes différents permettant ainsi leur recombinaison, sauf si les intermédiaires de recombinaison sont reconnus par les protéines MMR.

coiffe multiprotéique. En cas d'érosion importante, les télomères devenus trop courts peuvent avoir perdu cette coiffe de protection et donc devenir sensibles aux systèmes de réparation par recombinaison. Cependant, les répétions proximales «dégénérées », toujours présentes après l'érosion de la partie distale des télomères, pourraient être reconnues anormalement raccourcis sont sujets à bien plus de recombinaisons que ceux de taille «normale» [11]. Les interactions entre télomères anormalement raccourcis et système MMR ne semblent donc pas suivre des règles connues.

La fonction du système MMR, au niveau des télomères, ne se limite peut-être pas à empêcher leur recombinaison. De nombreux exemples d'organisation télomérique dans le noyau suggèrent l'existence d'interactions entre molécules d'ADN télomérique [12], qu'il s'agisse du regroupement des télomères [13] ou de la formation des boucles télomériques $(t$-loop) [14]. Le système MMR pourrait contrôler la fidélité de ces interactions et participer ainsi à l'agencement spatial des chromosomes. Enfin, en l'absence d'altérations identifiées des composants du système MMR, certaines cellules semblent présenter un processus ALT dont les mécanismes d'activation restent à identifier. Il est toutefois possible qu'une déficience transitoire du système MMR en soit la cause. En effet, on peut envisager l'existence, dans les cellules qui présentent un dysfonctionnement télomérique, d'une saturation du système de réparation qui serait due à un excès d'interaction télomérique ou à une accumulation de tout autre défaut reconnu par les protéines MMR. De la même façon, il est connu qu'un nombre anormal d'erreurs de réplication est responsable de l'inactivation du MMR chez le colibacille [15]. Bien que réalisés essentiellement dans le modèle de la levure, les travaux expérimentaux du laboratoire de V. Lundblad ont des implications tout à fait passionnantes dans le domaine de la cancérogenèse humaine. En effet, des déficiences du système MMR sont observées dans les syndromes de prédisposition aux cancers colo-rectaux non polyposiques (HNPCC pour hereditary non-polyposis colorectal cancer) et dans des cas sporadiques de carcinome du sein, du colon et d'autres tissus [16]. On sait que ce déficit est à l'origine d'une très forte augmentation du taux de mutations spontanées, favorisant ainsi l'apparition d'altérations d'oncogènes et de gènes suppres- 
seurs de tumeur. Les travaux de Lundblad suggèrent fortement que, parallèlement à cet effet, l'inactivation du système MMR provoque une augmentation de la survie cellulaire par activation du processus ALT, permettant ainsi l'immortalisation et la transformation maligne. En effet, l'introduction, dans le gène $M S H 2$ de levure, des mêmes mutations fauxsens que celles qui ont été identifiées chez des malades atteints du syndrome HNPCC, provoque une inhibition de la sénescence des levures. Il semble donc urgent d'établir d'éventuelles corrélations entre les tumeurs contenant des cellules ALT et la présence d'une anomalie du système MMR, ce qui nécessitera une meilleure caractérisation cytologique et moléculaire des cellules ALT. Ceci semble d'autant plus important que se développe actuellement tout un champ d'investigation portant sur la possibilité d'utiliser des inhibiteurs de la télomérase dans une finalité anticancéreuse $\left(\mathrm{m} / \mathrm{s} 2001, n^{\circ} 10\right.$, p. 1076). Les cellules cancéreuses maintenant la longueur de leurs télomères par le processus ALT pourraient alors échapper, au moins en partie, à ces nouvelles stratégies thérapeutiques, et l'association de molécules dirigées contre ce processus pourrait s'avérer très efficace.
1. Rudolph KL, Millard M, Bosenberg MW, DePinho RA. Telomere dysfunction and evolution of intestinal carcinoma in mice and humans. Nat Genet 2001; 28 : 155-9.

2. Hahn WC, Stewart SA, Brooks MW, et al. Inhibition of telomerase limits the growth of human cancer cells. Nat Med 1999; 5 : 1164-70.

3. Blackburn EH. The end of the (DNA) line. Nat Struct Biol 2000; $7:$ 847-50.

4. Shay JW, Bacchetti S. A survey of telomerase activity in human cancer. Eur J Cancer 1997; 33 : 787-91.

5. Bryan TM, Englezou A, Dalla-Pozza L, Dunham MA, Reddel RR. Evidence for an alternative mechanism for maintaining telomere length in human tumors and tumor-derived cell lines. Nat Med 1997; 3: 1271-4.

6. Dunham MA, Neumann AA, Fasching CL, Reddel RR. Telomere maintenance by recombination in human cells. Nat Genet 2000; 26: 447-50.

7. Rayssiguier C, Thaler DS, Radman M. The barrier to recombination between Escherichia coli and Salmonella typhimurium is disrupted in mismatch-repair mutants. Nature 1989; 342: 396-401. 8. Lundblad V, Blackburn EH. An alternative pathway for yeast telomere maintenance rescues est-1-senescence. Cell 1993; 73: 347-60.

9. Rizki A, Lundblad V. Defects in mismatch repair promote telomerase-independent proliferation. Nature 2001 ; 411 : 713-6.

10. Flint J, Bates GP, Clark K, et al. Sequence comparison of human and yeast telomeres identifies structurally distinct subtelomeric domains. Hum Mol Genet 1997; 6: 1305-13.

11. McEachern MJ, Iyer S. Short telomeres in yeast are highly recombinogenic. Mol Cell 2001; 7: 695-704.

12. Gilson E, Müller T, Sogo J, Laroche T, Gasser SM. RAP1 stimulates single- to double-strand association of yeast telomeric DNA: implications for telomere-telomere interactions. Nucl Acids Res 1994; 22 : 5310-20.
13. Palladino F, Laroche T, Gilson E, Axelrod A, Pillus L, Gasser SM. SIR3 and SIR4 proteins are required for the positioning and integrity of yeast telomeres. Cell 1993; 75 : 543-55.

14. Griffith JD, Comeau L, Rosenfield S, et al. Mammalian telomeres end in a large duplex loop. Cell 1999; 97 : 503-14.

15. Schaaper RM, Radman M. The extreme mutator effect of Escherichia coli mutD5 results from saturation of mismatch repair by excessive DNA replication errors. Embo J 1989; 8 : 3511-6.

16. Modrich P. Mismatch repair, genetic stability, and cancer. Science 1994; 266 : 1959-60.

\section{Michèle Brunori Éric Gilson}

Laboratoire de biologie moléculaire et cellulaire, UMR5665, Centre national de la recherche scientifique; École normale supérieure de Lyon, 46, allée d'Italie, 69364 Lyon Cedex 07, France.

\section{Alain Puisieux}

Centre d'oncologie génétique, Centre LéonBérard, 28, rue Laennec, 69008 Lyon, France.

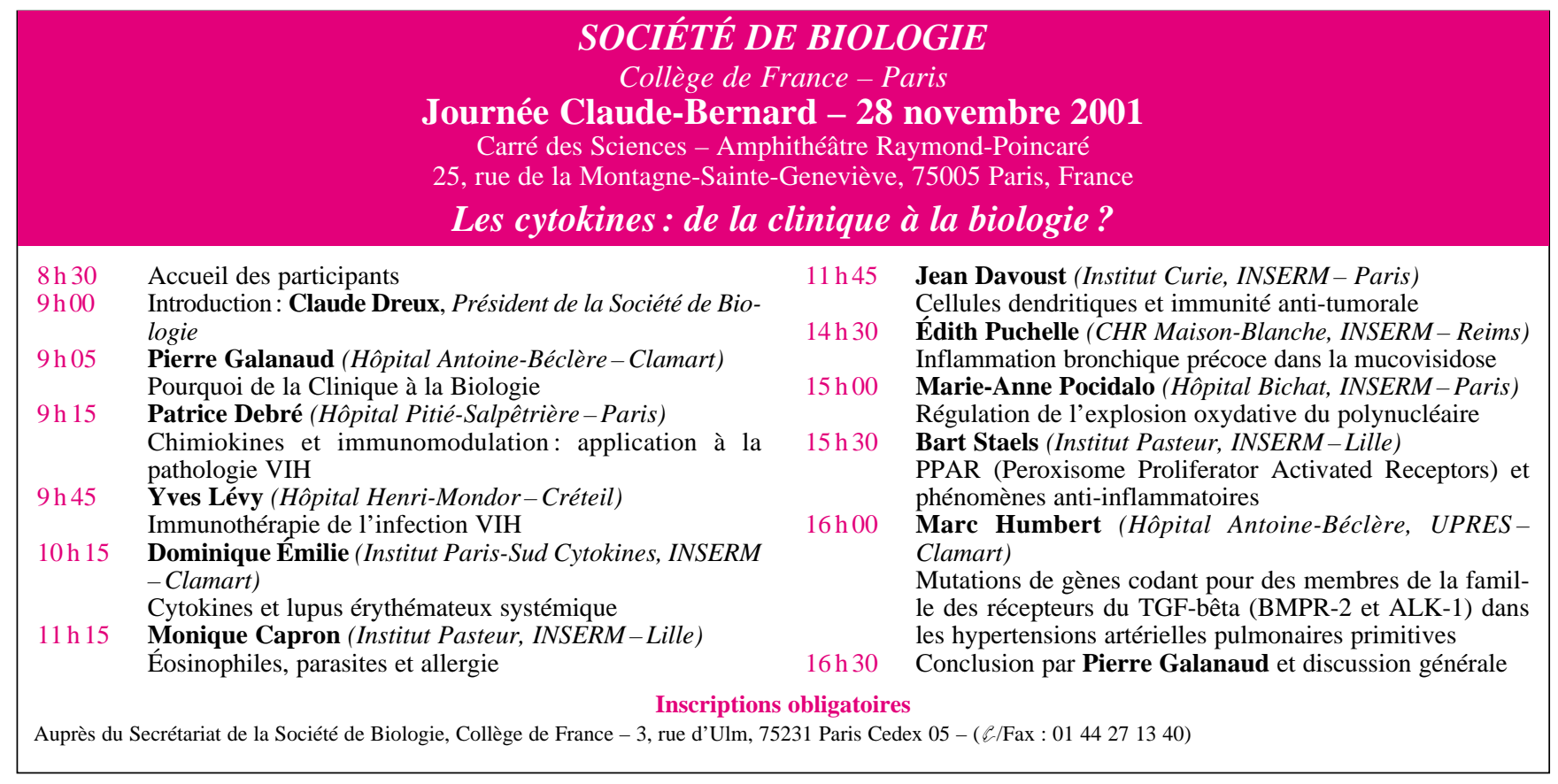



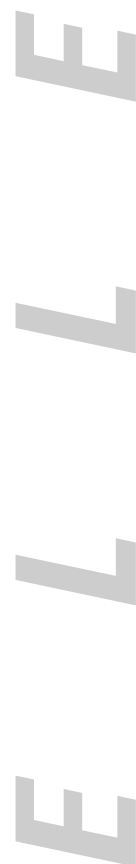